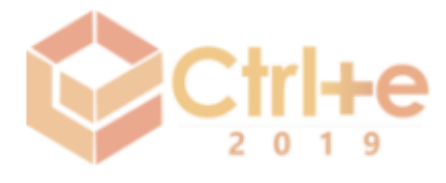

IV Congresso sobre Tecnologias na Educação (Ctrl+E 2019)

Recife, Pernambuco - Brasil

28 a 30 de agosto de 2019

\title{
Vamos Jogar Matemática: utilizando o RPG Maker para produzir um recurso educacional digital para o ensino de Matemática
}

\author{
Igor Eduardo de Lima Moreira1, Arianny de Sousa Lira² ${ }^{3}$, Darlene Alves Leitão ${ }^{2}$, \\ Jéssica de Alencar Rios ${ }^{1}$, Rafaela Gomes Nobreㅁ, Sarah Nidia Costa de Lima ${ }^{1}$, \\ Juscileide Braga de Castro ${ }^{12}$.
}

${ }^{1}$ Faculdade de Educação (FACED) - Universidade Federal do Ceará (UFC) - Fortaleza, CE - Brasil.

${ }^{2}$ Grupo de Pesquisa e Produção de Ambientes Interativos e Objetos de Aprendizagem PROATIVA. Instituto UFC Virtual

3Pós-Graduação em Ensino de Ciências e Matemática (PGECM) - Instituto Federal de Educação Tecnológica do Ceará (IFCE) - Fortaleza, CE - Brasil

\{arianny_sousa\}@hotmail.com, \{darlenealvesleitao,final.eduardo.7,jessic arios $449 \overline{6}$, rafaella.rgn, sarahnidia21\}@gmail.com, juscileide@virtual.ufc.

br

\begin{abstract}
This article aims to describe the digital educational resource (DER), Let's Play Math, created from RPG Maker, this being a free software that allows the creation of electronic RPG games. During its production, it was verified the need to learn mathematical concepts from the production and use of digital technologies, exploring competences related to protagonism, autonomy and authorship. The DER was developed to meet the number skills predicted by the $B N C C$ for the 4th year of Elementary School. Among the results, a new perspective for the teacher is emphasized, since it can create a game, promote its use or even build with the students.

Resumo. Este artigo tem como objetivo descrever o recurso educacional digital (RED), Vamos Jogar Matemática, criado a partir do RPG Maker, sendo este um software livre que permite a criação de jogos RPGs eletrônicos. Durante sua produção, verificou-se a necessidade da aprendizagem de conceitos matemáticos a partir da produção e uso de tecnologias digitais, explorando competências relacionadas ao protagonismo, à autonomia e à autoria. $O$ RED foi desenvolvido para atender às habilidades de números previstas pela BNCC para o $4^{\circ}$ ano do Ensino Fundamental. Ressalta-se como resultado uma nova perspectiva para o professor já que o mesmo poderá criar um jogo, promover sua utilização ou ainda, construir junto com os estudantes.
\end{abstract}

\section{Introdução}

Antes mesmo de iniciar uma vida escolar, a criança já tem seus primeiros contatos com a leitura, com a escrita e com os conceitos matemáticos. Quando a criança se depara com o conteúdo de aritmética na escola, por exemplo, muitas vezes já vivenciou, em seu 
convívio social, situações aditivas (ideias de juntar, separar, acrescentar, retirar, comparar, entre outras) e/ou multiplicativas (ideias de proporcionalidade, combinação, produto de medidas, partição, cotição, entre outras). Isto acontece, pois, as crianças trazem consigo, na bagagem, a sua própria aritmética pré-escolar [Vygotsky 1989].

Nesse âmbito, reconhecemos que a Matemática é uma das disciplinas que desempenha um papel decisivo em relação a vida escolar, pois une o raciocínio à muitas aplicações no cotidiano, auxilia no processo de resolução de problemas e funciona como instrumento de base para outras disciplinas curriculares, como a Física e a Química, por exemplo. Contudo, verifica-se que a escola explora muito pouco a bagagem cultural dos estudantes, pois costuma ensinar e avaliar a todos da mesma forma, ignorando a existência de diferentes competências: cognitivas, pessoais e sociais; e que estas, para serem desenvolvidas, precisam de abordagens diferenciadas [Morán 2015].

$\mathrm{O}$ aprendizado de matemática tem como alguma das finalidades, desenvolver o raciocínio lógico, a criatividade e a capacidade de desenvolver problemas. Miskulin (2003, p. 226) afirma que: “[...], a matemática deve ser mediada também por metodologias alternativas, em que o aluno em formação vivencie novos processos educacionais, plenos de sentido e relacionados com seus significados e valores. [...]". Nessa direção, observamos a importância de trabalhar a Matemática numa perspectiva diferenciada, colocando os estudantes como protagonistas da aprendizagem, o que pode promover a reflexão e a autonomia, e propiciando aulas participativas, em que todos possam realizar descobertas e produzir conhecimentos.

Competências como o protagonismo, a autonomia e a colaboração são previstas pela Base Nacional Comum Curricular (BNCC) e podem ser desenvolvidas a partir da exploração da cultura digital e da utilização de diferentes linguagens [Brasil 2017].

Neste viés, é importante ressaltar que a utilização de Tecnologias Digitais de Informação e Comunicação (TDIC), trabalhadas de forma integrada com os conteúdos de Matemática, podem desenvolver um maior interesse e envolvimento dos alunos diante do processo de aprendizagem. Portanto, atrelar o uso de tecnologias digitais com uma metodologia de ensino significava, pode estimular e proporcionar uma relação de interação entre os alunos e o professor [Leitão 2018].

É notório que o uso de tecnologias digitais tornou-se, de certa forma, algo necessário na sociedade atual. No ambiente escolar, o desenvolvimento de práticas que envolvam TDIC é cada vez mais comum. Nesse contexto, é importante salientar que o uso das tecnologias digitais não deve ser pensado como uma forma de transferência de informação, e sim como uma forma de transformar o ser em alguém ativo e dono do processo de construção do seu conhecimento [Papert 1985].

Diante das questões apresentadas, essa pesquisa tem como objetivo descrever o processo de produção, além de apresentar e analisar o recurso educacional digital (RED), Vamos Jogar Matemática. Esse RED foi construído com a ferramenta RPG Makerı para auxiliar o ensino de Matemática nos anos iniciais do Ensino Fundamental, podendo trazer contribuições ao educador por meio de aprendizagens significativas sobre os conceitos matemáticos explorados, bem como a sua aplicabilidade em sala de aula.

\footnotetext{
1 É um estilo de jogo que começou como "Jogos de representação" de mesa e tinha elementos básicos: Batalhas baseados em turnos, forte presença narrativa, teatralidade e temáticas que envolviam fantasia. Quando os jogos saíram da mesa e foram para os meios eletrônicos ganharam nova roupagem, mas os elementos base permanecem, em sua grande maioria, até os dias de hoje.
} 
Cabe ressaltar que este jogo foi desenvolvido de forma colaborativa, com o intuito de trabalhar com conjuntos numéricos e suas operações, tais como: Sistema de numeração decimal - leitura, escrita, comparação e ordenação de números naturais de até cinco ordens; Composição e decomposição de um número natural de até cinco ordens, por meio de adições e multiplicações por potências de 10; Propriedades das operações para o desenvolvimento de diferentes estratégias de cálculo com números naturais; Problemas envolvendo diferentes significados da multiplicação e da divisão: adição de parcelas iguais, configuração retangular, proporcionalidade, repartição equitativa e medida; Problemas de contagem e Números racionais: frações unitárias mais usuais.

O estudo tem como referência contribuições teóricas de Seymour Papert (1985), alinhando com outras abordagens teóricas atuais como as Metodologias Ativas, o que implica a exploração do protagonismo e da autoria, competências que são citadas na BNCC [Brasil 2017] e que podem vir a ser relacionadas com a formação docente.

Essa abordagem de pesquisa, assemelha-se com os trabalhos desenvolvidos por Fernandes et al (2018) e por Castro et al (2017). Ambos trabalhos tratam de produção de recursos educacionais digitais para o Ensino de Matemática.

Em seu trabalho, Castro et al (2017) apresentam o Design Thinking como um processo de construção de RED. Este processo foi desenvolvido junto a estudantes de Pedagogia, como forma de fazê-los refletir sobre conceitos, teorias e metodologias que poderiam estar envolvidos nos conteúdos contemplados por cada RED. A pesquisa apresenta evidências de que esta abordagem contribuiu para "a troca de experiências e saberes, a reflexão, a construção, a pesquisa, a análise e a formulação de métodos próprios para resolver situações matemáticas" [Castro et al 2017, p. 489].

Fernandes et al (2018) apresentam um RED para o ensino de Estatística nas escolas. A construção deste RED foi pensada considerando o processo de aprendizagem de conteúdos curriculares matemáticos com a utilização de tecnologias digitais. Sendo assim, além dos conteúdos matemáticos, foram verificados elementos como personagens e narrativa, como forma de proporcionar um contexto adequado para o trabalho com gráficos.

Ainda que estas pesquisas apresentem processos semelhantes ao que se propõe neste artigo, nenhuma delas utilizou a ferramenta RPG Maker como uma possibilidade de produção de RED. Neste sentido, na próxima seção, discorre-se sobre o contexto e o desenvolvimento do jogo digital. Em seguida, apresenta-se o recurso e a análise realizada, seguida das considerações finais.

\section{Contexto e Desenvolvimento}

Este artigo caracteriza, descreve e analisa o RED, Vamos Jogar Matemática, desenvolvido no decorrer da disciplina de Ensino de Matemática do curso de Pedagogia da Faculdade de Educação (FACED) da Universidade Federal do Ceará (UFC), durante o semestre 2018.2.

O desenvolvimento deste RED foi proposto como uma das atividades da disciplina, como forma de explorar conceitos, teorias e metodologias relacionadas ao Ensino de Matemática nos anos iniciais do Ensino Fundamental. Para isso, utilizou-se como abordagem o Design Thinking, no qual contempla métodos e processos para resolução de problemas, divididos em 4 etapas não lineares: 
[1] imersão - nesta etapa toma-se conhecimento da situação geral, do tipo de problema que se deseja resolver, além das necessidades de todas as partes interessadas no produto final, em especial o cliente-alvo; [2] análise e síntese - considera-se, nesta etapa, os dados recolhidos durante o processo de imersão e as especificações do problema a ser resolvido são delimitadas; [3] ideação etapa em que busca-se a síntese do problema, sendo lançadas propostas de solução, que são anotadas, filtradas e reunidas até se chegar numa proposta final; e [4] prototipação e testes, onde a partir da proposta final se gera um produto puramente funcional que então será testado na sua capacidade de resolver o problema antes de ser finalizado [Castro et al 2017, p. 486-487].

Neste contexto, ao longo do processo, analisou-se diferentes soluções para a narrativa proposta no jogo, assim como, para os conceitos matemáticos trabalhados. A unidade temática números foi escolhida por meio de sorteio, sendo considerado, para isso, as cinco unidades de matemática: números, álgebra, geometria, grandezas e medidas e probabilidade e estatística [Brasil 2017].

O RED foi desenvolvido considerando as habilidades a serem trabalhadas na unidade de números para os estudantes do $4^{\circ}$ ano do Ensino Fundamental, contemplando nove habilidades, conforme quadro 1 , a seguir.

Quadro 1. Habilidades da BNCC exploradas no jogo digital: Vamos Jogar Matemática

(EF04MA01) Ler, escrever e ordenar números naturais até a ordem de dezenas de milhar

(EF04MA02) Mostrar, por decomposição e composição, que todo número natural pode ser escrito por meio de adições e multiplicações por potências de dez, para compreender o sistema de numeração decimal e desenvolver estratégias de cálculo

(EF04MA03) Resolver e elaborar problemas com números naturais envolvendo adição e subtração, utilizando estratégias diversas, como cálculo, cálculo mental e algoritmos, além de fazer estimativas do resultado

(EF04MA04) Utilizar as relações entre adição e subtração, bem como entre multiplicação e divisão, para ampliar as estratégias de cálculo

(EF04MA05) Utilizar as propriedades das operações para desenvolver estratégias de cálculo

(EF04MA06) Resolver e elaborar problemas envolvendo diferentes significados da multiplicação (adição de parcelas iguais, organização retangular e proporcionalidade), utilizando estratégias diversas, como cálculo por estimativa, cálculo mental e algoritmos

(EF04MA07) Resolver e elaborar problemas de divisão cujo divisor tenha no máximo dois algarismos, envolvendo os significados de repartição equitativa e de medida, utilizando estratégias diversas, como cálculo por estimativa, cálculo mental e algoritmos

(EF04MA08) Resolver, com o suporte de imagem e/ou material manipulável, problemas simples de contagem, como a determinação do número de agrupamentos possíveis ao se combinar cada elemento de uma coleção com todos os elementos de outra, utilizando estratégias e formas de registro pessoais

(EF04MA09) Reconhecer as frações unitárias mais usuais $\left(\frac{1}{2}, \frac{1}{3}, \frac{1}{4}, \frac{1}{5}, \frac{1}{10}\right.$ e $\left.\frac{1}{100}\right)$, como unidades de medida menores do que uma unidade, utilizando a reta numérica como recurso

Vale ressaltar que, ainda que o RED tenha sido desenvolvido para atender as habilidades de números previstas para o $4^{\circ}$ ano do Ensino Fundamental, a aplicação do recurso adapta-se igualmente a outros níveis escolares. 
Escolhemos a produção de um RED com as características de um jogo de RPG, pois, neste tipo de jogo, o usuário é convidado a imergir na narrativa do jogo, devido a interação necessária nos diferentes cenários. Ademais, pensamos em tornar a disciplina de matemática mais significativa, fazendo com que o estudante associe o conhecimento adquirido à sua vida cotidiana, já que, ao mesmo tempo, a utilização do jogo funciona como um meio de transição do pensamento concreto para o abstrato, facilitando o desenvolvimento do raciocínio lógico.

Sendo assim, verificamos que o RPG Maker VXace poderia ser usado como uma ferramenta para o desenvolvimento do jogo que teríamos que produzir, pois possui recursos para criação de funções gráficas, além de opções para acrescentar física aos objetos, trilhas sonoras, entre outras ações. Embora o programa seja comercial, é possível utilizá-lo gratuitamente baixando a distribuição RPG Maker VXace Lite. Essa versão se mostra extensa o suficiente para permitir a criação de projetos simples e divertidos.

Essa ferramenta é, como o nome sugere, utilizada para criar jogos do gênero RPG (Role Playing Game) eletrônicos. A primeira versão da ferramenta foi criada pela empresa japonesa ASCII, as versões posteriores que foram desenvolvidas assumidas pela, também japonesa, Enterbrain, incluindo a que foi utilizada na construção de nosso jogo. $\mathrm{O}$ Software foi criado com o objetivo de tornar popular a criação de jogos de RPG eletrônico, isso porque é possível criar jogos de grande dimensão, sem grandes dificuldades, não necessitando ter um grande conhecimento em linguagem de programação [Rosa; Maltempi 2003].

Além disso, é possível perceber que o programa tem por base e inspirações nos RPG's clássicos, tais como Chrono Trigger e Final Fantasy III, da $4^{\mathrm{a}}$ Geração de games, como o Super Nintendo, que foram lançados na década de 90, o que contribuiu para uma popularização do uso do programa, uma vez que não é necessário ter um computador ou hardware potente para poder produzir os recursos do RPG Maker. Cabe ressaltar, que a sua utilização em processos de ensino e aprendizagem é válido, uma vez que é um tipo de mídia que tem linguagem e mecânica acessíveis para públicos diversos, inclusive alunos em idade escolar.

A seguir, em resultados e discussões, apresentar-se-á e analisar-se-á o RED Vamos Jogar Matemática.

\section{Resultados e discussões}

A apresentação e a análise do RED serão divididas em duas seções. A primeira, apresentará uma descrição do RED produzido, explicitando os conceitos matemáticos abordados. Na segunda, será feita uma análise das contribuições e limitações do RED.

\subsection{O RED Vamos Jogar Matemática}

No projeto do RPG Maker VXace, do jogo Vamos Jogar Matemática2, a interface de programação apresentada na Figura 1, possui 12 cenários (Vila, Casa, Parquinho, Corredores da Escola, entre outros), porém, em apenas 6 ambientes tem desafios que buscam trabalhar habilidades do ensino de Matemática.

2 Disponível para download, em formato WinRAR, compartilhado no Google Drive, em: https://drive.google.com/file/d/1w7A41OFviSkjPV-m66eOe510cfUZZEgk/view?usp=drivesdk. Direitos autorais para a música do jogo Vamos Jogar Matemática: Chrono Trigger - Jogo RPG Eletrônico desenvolvido pela Square Co. 


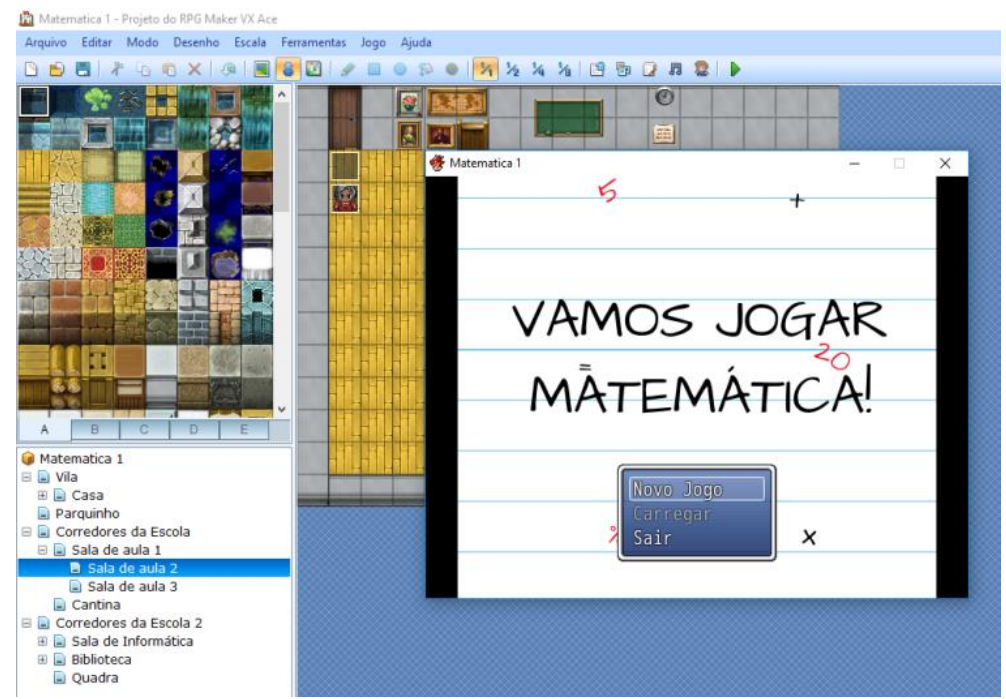

Figura 1. Projeto do RPG Maker VXace: jogo VAMOS JOGAR MATEMATICA

O propósito é vivenciar a história para além do game player. Cada personagem possui uma pequena fala, direcionando o usuário as ações do jogo, e as mesmas serão executadas a partir da utilização do controle de ação, que é o comando Z. Os eventos de gatilho e desafios iniciam por meio do comando Z, e nesses 6 ambientes apresentados no Quadro 2, é o local onde ocorrerá tais desafios.

Quadro 2. Ambientes e Ações: Desafios - jogo VAMOS JOGAR MATEMATICA

\begin{tabular}{|l|l|}
\hline \multicolumn{1}{|c|}{ AMBIENTES } & \multicolumn{1}{|c|}{ AÇÃO NECESSÁRIA } \\
Sala de Aula & $\begin{array}{l}\text { Terá um cartaz de aviso, e ao tocar o sinal de intervalo, Kaio senta em sua carteira } \\
\text { e a partir desse momento, apertando o comando Z ele será teletransportado ao } \\
\text { desafio; }\end{array}$ \\
\hline Sala de Informática & $\begin{array}{l}\text { O personagem será direcionado ao computador localizado no fundo da sala, e ao } \\
\text { ativar o comando Z, terá acesso às informações do próximo desafio; }\end{array}$ \\
\hline Cantina & $\begin{array}{l}\text { No espaço da cantina o personagem irá interagir com a merendeira para descobrir } \\
\text { os passos para próximo desafio; }\end{array}$ \\
\hline Biblioteca & $\begin{array}{l}\text { Na biblioteca, Kaio estará à procura do bibliotecário, o mesmo lhe direcionará } \\
\text { ao próximo desafio; }\end{array}$ \\
\hline Pátio & $\begin{array}{l}\text { O personagem irá se deparar com o monstro do pátio ao explorar o local até a } \\
\text { caixa de areia, e tendo como objetivo derrotá-lo para avançar aos próximos } \\
\text { desafios. }\end{array}$ \\
\hline Quadra & $\begin{array}{l}\text { Na quadra se encontra o último desafio, e para desvendá-lo o personagem deverá } \\
\text { interagir com o professor de Educação Física, que se encontra no centro da } \\
\text { quadra. }\end{array}$ \\
\hline
\end{tabular}

Temos como personagem o Kaio, o avatar que será utilizado pelos usuários do jogo. Kaio está muito ansioso por conta de um evento cultural na escola, a narrativa inicial vai se desenvolvendo em um mundo de fantasia projetado por ele em um sonho.

Inicialmente Kaio dormirá e ao acordar irá conversar com seus pais sobre o evento cultural, após o diálogo, o mesmo se dirige a escola. Ao conversar com seus colegas da escola, ele será direcionado a seu primeiro desafio, que é vencer o monstro do pátio, resolvendo um questionamento sobre combinações: quantas combinações você pode fazer para que todos os 5 amigos brinquem com você na gangorra? Esta situação tem como referência a habilidade EF04MA08, como apresentada no Quadro 1. 
Concluindo o desafio de forma assertiva, Kaio passará pelo corredor da escola e encontrará na primeira porta, a Sala de Aula, onde a atividade proposta pela professora Marielle é ordenar os números até a quarta ordem, habilidade EF04MA01, tendo como representatividade de números os próprios alunos. Outro local, apresentado na segunda porta do corredor, é a Cantina, onde a merendeira conversa com Kaio, e o questiona com o seguinte desafio: distribua 5 décimos de frutas para cada aluno, qual será a representação fracionária correta? - habilidade EF04MA09.

No próximo corredor encontramos a Sala de Informática (Figura 2), o desafio presente nesse cenário faz com que Kaio e seus colegas de classe vençam com a ajuda de Norton, o antivírus, uma batalha de composição e decomposição de números, em que o número 8653 estava escrito por meio de adições e multiplicações por potências de dez, explorando a habilidade EF04MA02.

O desafio da Sala de Informática apresenta uma narrativa interdisciplinar, tendo em vista, que as questões apresentadas agem de forma integrada com o ensino, ao apresentar uma batalha contra um vírus de computador, reforçando situações que envolvem vivências no ambiente escolar, desde os espaços internos e externos da escola. Os desafios consolidaram a validação da narrativa e das atitudes interdisciplinares como caminho para o saber compartilhado e construído, demonstrando ainda que o Vamos Jogar Matemática apresenta perfil para ser uma ferramenta adequada no processo educativo, desde que sejam aproveitados seus ambientes, cenários e desafios.

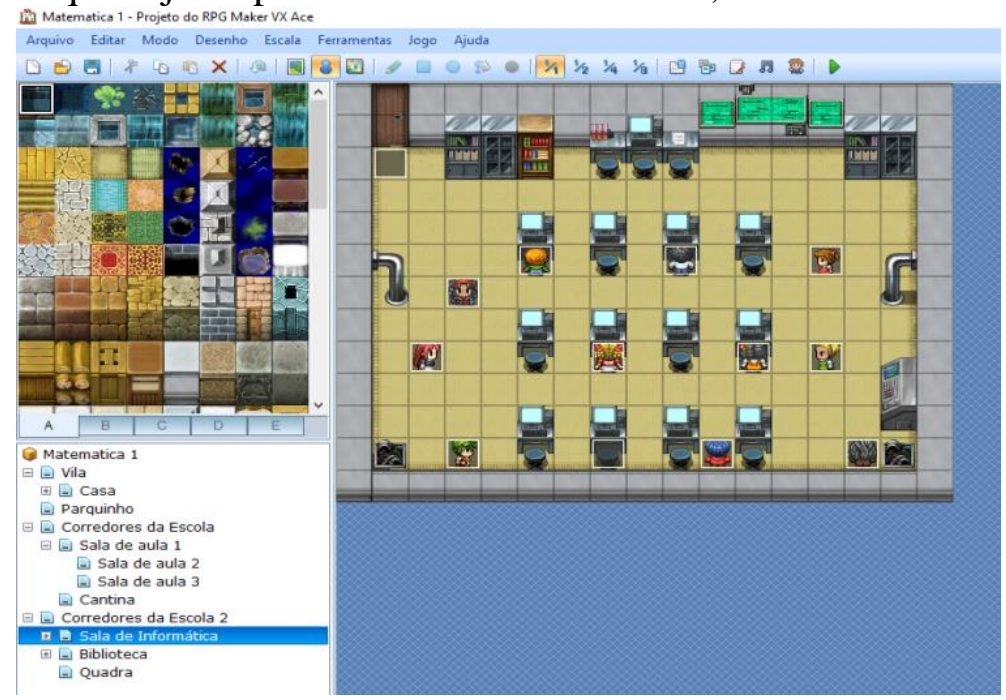

Figura 2. Sala de Informática: jogo VAMOS JOGAR MATEMATICA

$\mathrm{Na}$ Biblioteca, em conversa com o bibliotecário, Kaio percebe que um espírito guardião dos livros vem assombrando o local. O guardião o questiona sobre a operação 3 x 7 x 0, habilidade EF04MA04. Precisamos levar em consideração que os desafios apresentam mais de uma habilidade da BNCC, pois devemos utilizar as propriedades das operações para desenvolver estratégias de cálculo, utilizando assim a habilidade EF04MA05.

E por fim, a Quadra, onde seu professor de Educação Física, que encontra-se no meio da quadra, o orientará sobre a atividade proposta que envolve localização, já que a quantidade de passos é descrita a partir da resolução de operações de soma e subtração, habilidade EF04MA03. Obtendo como resposta: 4 passos para baixo, 1 passo a direita, 11 passos para a esquerda e 5 passos para cima, e assim encontrando o prêmio final. Ao final desses desafios, Kaio retorna ao seu quarto, e se questiona: Foi tudo um sonho, como 
assim? Mas, por fim, deseja que consiga resolver todas as situações problemas no evento cultural da escola, assim como conseguiu no seu sonho.

Por ser um jogo de estilo RPG, foi possível por meio das caixas de diálogo presentes nas interações entre os personagens, momentos de batalhas, eventos e criar situações em que os problemas propostos estivessem coerentes com o público alvo e com os objetivos de aprendizagem que desejamos alcançar.

Na sessão a seguir, apresentaremos as contribuições e limitações do RED, tendo como implicações o papel do professor e abordando a teoria construcionista como embasamento teórico.

\subsection{Contribuições e limitações do RED}

Vamos Jogar Matemática, apresenta uma interação constante do jogador com os elementos presentes no recurso, visto que para avançar de fase é necessário descobrir e concluir os desafios apresentados. Deste modo, o jogador deverá desbravar todos os ambientes, buscar dicas e vencer batalhas, mediante acerto de questões de Matemática.

A utilização do RED em sala de aula traz como proposta que os alunos explorem o jogo, resolvam as situações problema, passando por todas as fases até o jogo ser finalizado. O recurso será utilizado como uma estratégia, com o intuito de apresentar um material de design digital mais atraente ao aluno, envolvendo-o nos desafios ao mesmo tempo em que apresenta situações que envolvam problemas matemáticos. O professor estará envolvido nas atividades com a turma, auxiliando em quaisquer dúvidas que possam surgir e no processo de ensino. Caso não existam máquinas suficientes para cada aluno, o jogo poderá ser explorado em duplas ou trios.

Essa concepção pode ser associada com as competências gerais 2 e 5 definidas pela BNCC [Brasil 2017]:

Competência 2: Exercitar a curiosidade intelectual e recorrer à abordagem
própria das ciências, incluindo a investigação, a reflexão, a análise crítica, a
imaginação a criatividade, para investigar causas, elaborar e testar hipóteses,
formular e resolver problemas e criar soluções (inclusive tecnológicas) com
base nos conhecimentos das diferentes áreas.[...] Competência 5:
Compreender, utilizar e criar tecnologias digitais de informação e
comunicação de forma crítica, significativa, reflexiva e ética nas diversas
práticas sociais (incluindo as escolares) para se comunicar, acessar e
disseminar informações, produzir conhecimentos, resolver problemas e
exercer protagonismo e autoria na vida pessoal e coletiva. (BRASIL, 2017, sn,
grifo do autor)

Nesse contexto, unindo tecnologia com a educação, procuramos nos fundamentar na teoria pedagógica do construcionismo [Papert 1985], que relaciona as tecnologias com a educação. As tecnologias digitais têm chegado às escolas, a partir da disposição de ferramentas como computadores e dispositivos portáteis. Essa conexão pode propiciar experiências relevantes, possibilitando o desenvolvimento de projetos interdisciplinares, a geração de dados em tempo real e a interação [Castro e Castro-Filho 2012].

O construcionismo corrobora com o uso da tecnologia, com ênfase, na utilização do computador tendo o usuário como centro do processo de construção do aprendizado. Entretanto, é importante salientar que o recurso da pesquisa também apresenta ações instrucionistas, todavia não invalida a perspectiva geral do jogo, que permite ao usuário, explorar vários ambientes e cenários, interagir com os elementos presentes e buscar compreender as situações com o objetivo de avançar os desafios. 
O RED, sendo jogo de estilo RPG, no âmbito do ensino de matemática assume uma nova perspectiva para o professor já que o mesmo poderá criar um jogo, pois a linguagem de programação nos permite esse procedimento, ou construir de forma colaborativa juntamente com os alunos, apresentando assim uma abordagem construcionista e, a partir disso, promover sua utilização com os alunos de forma apenas instrucionista com foco na resolução de situações problemas.

É necessário entender que sua elaboração ocorreu a partir de uma plataforma básica de elaboração de jogos, não se fazendo necessário conhecimento aprofundado sobre programação, apresentando facilidade e rapidez na criação de protótipos. Outra vantagem são os modelos pré-configurados de mapas, personagens e itens de cenários, presentes na versão, possuindo também gerenciadores de plugins.

No entanto, conseguimos perceber no âmbito de estruturação que este possui algumas limitações específicas, tais como as questões não serem randômicas, os personagens serem limitados, a qualidade dos gráficos é baixa, a parte física e o sistema de mensagens é limitado, o gameplay e interface não apresentam muitas opções de personalização, os ambientes criados não possuírem uma maior diversidade, e se for necessário utilizar outros ambientes ou expandir as opções dos personagens e cenários, há a necessidade de baixar outras extensões do programa que são pagas e com exceção da versão MV, o RPG Maker produz jogos apenas para o Windows.

\section{Considerações Finais}

Nesse artigo apresentamos e analisamos o RED, Vamos Jogar Matemática, no qual é detalhado como o RED funciona, a importância do seu uso no ensino e aprendizado de conceitos matemáticos sobre conjuntos numéricos e suas operações, sua ligação com as TDIC, suas contribuições e limitações, assim como, reconhecemos o construcionismo como subsídio a aprendizagem por meio da construção de jogos digitais.

O desenvolvimento do RED foi um processo que viabilizou aprendizagens de conceitos matemáticos, como também, de reflexões e análises metodologias envolvendo as TDIC. Nesta perspectiva ressaltamos a importância desse processo de construção de mídias digitais pelos estudantes da licenciatura como práticas que possibilitem essa reflexão acerca da inserção das TDIC nas práticas docentes.

Desse modo, conclui-se que o processo de construção dos recursos, possibilitou o desenvolvimento de uma nova percepção dos participantes perante o uso de TDIC no ambiente educacional e diante das abordagens metodológicas de ensino. Vale ressaltar, que a Base Nacional Comum Curricular apresenta em uma de suas competências, que a cultura digital, está ligada à compreensão, à criação e o uso de tecnologias digitais, como forma de produzir conhecimento, resolver problemas, dentre outros [Brasil 2017].

Por fim, espera-se que outras questões possam ser abordadas e discutidas com novos olhares sobre o uso de TDIC, como no caso do recurso desta pesquisa, podendo assim trazer futuras contribuições para o processo de ensino e de aprendizado. Possibilitando que as mídias desenvolvidas, sejam formas de reflexão dos conceitos, metodologias e teorias, e que estas sejam aplicadas e validadas no ambiente escolar.

\section{Referências}

Brasil, Ministério da Educação (2017). "Base Nacional Comum Curricular”. Disponível em <http://basenacionalcomum.mec.gov.br/> Acesso em: 31 Mar. 2018. 
Castro, J. B.; Soares, I. O.; Vianna, E. P. N.; Sousa, J. S. "Lógica Criativa: a construção de mídias digitais para o ensino de matemática". In: Anais dos Workshops do VI Congresso Brasileiro de Informática na Educação (WCBIE 2017). VI Congresso Brasileiro de Informática na Educação (CBIE 2017). Disponível em: <http://www.brie.org/pub/index.php/wcbie/article/view/7431/5227>. Acesso em: 23 mar. 2019.

Castro, J. B.; Castro-Filho, J. A. (2012) 'Projeto Um Mundo de Informações: Integração de Tecnologias Digitais ao Currículo Escolar'. In: Congresso Brasileiro de Informática na Educação, 1., 2012, Rio de Janeiro. Anais... Rio de Janeiro: SBC.

Fernandes, K.; Sousa, G.; Kelly, F.; Lima, G.; Leitão, D.; Souza, D.; Castro, J.. "Tecnologias Digitais e o Ensino de Estatística: explorando a Estatística a partir de um recurso educacional digital”. 2018. Mostra de Práticas de Informática na Educação. VII Congresso Brasileiro de Informática na Educação (CBIE 2018). Disponível em: <http://dx.doi.org/10.5753/cbie.wcbie.2018.357>. Acesso em: 28 fev. 2019.

Miskulin, R. G. S. “As possibilidades didático-pedagógicas de ambientes computacionais na formação colaborativa de professores de Matemática. In FIORENTINI, Dário. "Formação de Professores de Matemática". Campinas-SP. Mercado de Letras, 2003.

Papert, Seymour. "Logo: Computadores e Educação". 3 $3^{\mathrm{a}}$ ed. São Paulo: Editora brasiliense s.a. 1985.

Rosa, M.; Maltempi, M. V. "RPG MAKER: uma proposta para unir jogo, informática e educação matemática”. 2003. 20 f. TCC (Graduação) - Curso de Matemática, Universidade Estadual Paulista, Rio Claro, 2003. Disponível em: <http://www.rc.unesp.br/igce/demac/maltempi/Publicacao/Rosa-Maltempisipem03.pdf >. Acesso em: 30 nov. 2018.

Leitão, D. A. "Um olhar pedagógico sobre o processo de construção de recursos digitais de matemática com Scratch”. Fortaleza 2018. 54f. Monografia, Curso de Pedagogia Universidade Federal do Ceará (UFC)

Morán, J. "Mudando a Educação com metodologias ativas. In: Convergências Midiáticas, Educação e Cidadania: aproximações jovens". Vol. II. Coleção Mídias Contemporâneas. SOUZA, Carlos Alberto e MORALES, Ofelia Elisa Torres (Orgs.). PG: Foca FotoPROEX/UEPG, 2015. Disponível em: <http://www2.eca.usp.br/moran/wp-content/uploads/2013/12/mudando_moran.pdf> Acesso em: 05 set. 2018.

Vygotsky, L. S. “Pensamento e linguagem”. São Paulo: Livraria Martins Fontes, 1989. 